GUT MICROBIOTA

\title{
Targeting of specific microbial species mitigates colitis
}

Tungstate treatment prevents the inflammation-associated dysbiotic expansion of Enterobacteriaceae in mouse models of colitis, according to a new study published in Nature. The work represents proof of concept for the modulation of specific intestinal microbiota species.

Perturbations to the intestinal microbiota are associated with a number of inflammatory diseases, including IBD. The complexity of the system makes defining causality difficult, although microbiota transfer experiments in mice show that dysbiosis can exacerbate IBD in genetically susceptible hosts. "In my lab, we were interested in finding out whether dysbiosis could occur as a consequence of inflammation, and whether dysbiosis was a bystander effect or a driver of disease," explains author Sebastian Winter.

In earlier work, Winter and colleagues found that microbial molybdenum-cofactor-dependent metabolic pathways - notably nitrate respiration and formate dehydrogenation - are enriched in inflammation-associated dysbiosis and contribute to increased levels of Enterobacteriaceae, such as Escherichia coli. "Both metabolic pathways only operate in the inflamed gut and are not used by Enterobacteriaceae in the healthy gut," says Winter.

Winter and colleagues reasoned that administration of tungsten, which inserts in place of molybdenum to inhibit the molybdopterin cofactor required by both nitrate reductases and formate dehydrogenases, could be used to specifically inhibit E. coli overgrowth in IBD models. In a mouse model of colitis induced by dextran sulfate sodium (DSS), the researchers showed that administration of tungstate abolished the overrepresentation of gut microbial nitrate respiration and formate dehydrogenation pathways induced by DSS treatment. Notably, tungstate was highly selective; only

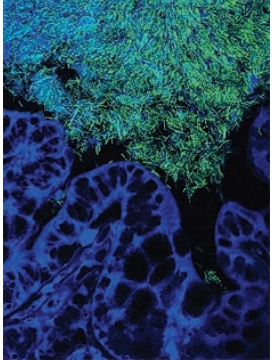

Bacteria (green) in the mouse large intestine (blue). Image courtesy of S. Winter.
Enterobacteriaceae abundance was substantially affected, and levels in healthy mice were not altered.

Tungstate administration reduced DSS-induced mucosal inflammation, as defined by caecal and colonic histology and expression of proinflammatory markers. Validating their model, the investigators found that tungstate also improved markers of inflammation in an $\mathrm{IllO}^{-1-}$ mouse model of colitis. Tungstate did not improve DSS-induced colitis in germ-free mice, suggesting a mechanism dependent on the modulation of the gut microbiota.

"I am particularly excited about this study because it demonstrates that a fundamental understanding of bacterial physiology can facilitate the rational development of strategies to manipulate the gut microbiota," enthuses Winter. "Furthermore, our data indicate that the bloom of Enterobacteriaceae that goes hand in hand with inflammation is not a mere bystander effect, but exacerbates mucosal host responses."

Hugh Thomas

ORIGINAL ARTICLE Zhu, W. et al. Precision editing of the gut microbiota ameliorates colitis. Nature https://doi.org/10.1038/ nature25172 (2018) 